\title{
PC based Heart Sound Monitoring System
}

\author{
Arathy $\mathrm{R}$ \\ Assistant Professor in \\ Electronics and biomedical \\ Engineering \\ Model Engineering College \\ Thrikkakara, Cochin
}

\author{
Gowriprabha V \\ PGET-D\&D,Skanray \\ Healthcare pvt Itd \\ plot\#360, KIADB Indl.Area, \\ Hebbal, Mysore
}

\author{
Vysakh V \\ Assistant Professor in Safety \\ Engineering \\ Toc $\mathrm{H}$ Institute of Science and \\ Technology \\ Arakunnam, Cochin
}

\begin{abstract}
Heart diseases caused by the heart and blood vessels disorders are world's largest killers. Hence early detection and monitoring of heart abnormalities is essential for diagnosis and effective treatment of heart diseases. This project aims to develop a low cost portable digital stethoscope. The primary function of this system is to work exactly like a normal stethoscope, but having an added advantage of not being limited by the length of the tubing with the help of wireless transmission. At the same time many people can listen to the stethoscope output which is normally impossible with an ordinary stethoscope. It also provides data to visually chart results by connecting to an off-instrument display such as a computer monitor so that further processing and diagnosis of abnormal phonocardiogram will be easy. For real time monitoring and for diagnosis LabVIEW application software is being used and finally a classification study is done using MATLAB and naïve bayers classifier. This advanced functionality can increase the physician's diagnostic capability.
\end{abstract}

\section{General Terms}

PCG, murmurs, artifacts, extrasystole, normal, FFT, naive bayers classification, decision tree classification

\section{Keywords}

Heart Sound, Digital stethoscope, PCG classification, Wireless stethoscope

\section{INTRODUCTION}

A stethoscope is used mainly to record heart sounds in the body. Stethoscopes are also a primary aid to diagnosis various heart abnormalities. Though the electrocardiograph was a significant breakthrough in the field of medicine, it only showed the chronological sequence of mechanical events occurring in the heart as a waveform representation. It did not take into consideration, the Individual frequency components of the heart signal, an analysis of which would give an accurate diagnosis of the working of the heart. This led to the development of the phonocardiograph (PCG). The stethoscope system when interfaced with a PC is capable of analyzing the heart sounds and identifying abnormalities in the heart sound and its classification. Storage of heart sound for later analysis is also possible. This advanced functionality increases the physician's diagnostic capability, and such a PCG is not still available in most hospitals. Acoustic stethoscope can be changed into a digital stethoscope by inserting an electrets microphone into its chest piece. This project presents the design and development of a low cost portable wireless/tubeless digital stethoscope which can be used by the physician for monitoring the patient from a distance. Also, a classification study is done to classify PCG signal as normal and abnormal.

\section{MOTIVATION}

In India significant percentage of the people who die due to cardiovascular diseases belong to rural part. The major reasons are lack of awareness, lifestyle, and the cost factor. While the first two reasons are more applicable in urban India, the third is a pain point for rural areas where there are no costeffective ways available for an early diagnosis of heart diseases. There are different tests and investigations used to diagnose heart disease. The tests doctor chooses depending upon risk of heart disease, history of heart problems and the symptoms one might have. But the initial diagnosis can be done using the stethoscope used for auscultation. For many years healthcare professionals would listen quietly to patient's internal organs so they could diagnose from specific sounds of these organs. But it requires expert (Doctor/Paramedics) for an accurate diagnosis of the working of the heart .Also, reach of current systems is limited by the length of the tubing and hence requires a wireless transmission system. Real time monitoring at affordable cost is still not available. Storage of heart sounds for later analysis not available in most hospitals. PCG gives the recording of the heart sound, but it is not widely seen in the market and its cost is very high. Hence there requires a low cost PC based heart sound monitoring system which offers the advantage of electronic, digital, recording and wireless stethoscopes.

\section{PHONOCARDIOGRAM AND ITS SIGNIFICANCE}

The instrument used for recording the sounds connected with the pumping action of heart is known as phonocardiograph and recording which is available is phonocardiogram. The auscultation of heart sound reveals valuable information about the functional integrity of the heart to the clinician. More information becomes available when clinician compares the temporal relationships between the heart sounds and the mechanical, electric events of the cardiac cycle. Developing such kind of diagnostic skill through either a conventional acoustic stethoscope or an electronic one is itself a very special skill, and it may take years to acquire, in particular recognizing the actual heart sounds and the heart murmurs. . Hence, recording of the heart sound in the form of the waveform display called Phonocardiogram (PCG) has been developed over the years to visually inspect the heart sound for diagnosis. The heart sounds are due to mechanical events such as movements of heart wall, closure of walls and due to turbulence and leakage of blood flow [1].These heart sounds can be used as a primary screening technique for diagnosing different heart disorders. It has been seen that the heart sounds 
produced by healthy hearts are remarkably identical and abnormal sounds always correlate to specific abnormalities.

A normal PCG recording consists of four heart sound components. They are First heart sound (S1), Second heart sound (S2), third heart sound (S3) and fourth heart sound (S4). Each heart sound cycle consists of two major periods called systole and diastole. Heart murmurs are high-frequency signals that occur in the systole or/and diastole intervals [2].

\subsection{S1 Sound}

$\mathrm{S} 1$ sound is caused by the closure of mitral and tricuspid valves. It corresponds to $\mathrm{R}$ wave of the ECG signal. It has longer duration with a range 50 to $100 \mathrm{~ms}$. S1 sounds have lower frequency and the frequency range is 30 to $100 \mathrm{~Hz}$. S1 has greater intensity when compared with second heart sound S2. S1 is common in normal and abnormal heart sounds. It is loud with mitral stenosis and diminishing with mitral regurgitation [3].

\subsection{S2 Sound}

It is caused due to the closure of aortic and pulmonary valves. It shows the end of systole and the beginning of diastole. S2 has higher pitch when compared with $\mathrm{S} 1$. The frequency is above $100 \mathrm{~Hz}$ and duration range is 25 to $50 \mathrm{~ms}$. Split in S2 is seen in patients with atrial septal defect; pulmonic stenosis and right bundle branch block [3].

\subsection{S3 Sound}

It is caused by the rapid ventricular filling in early diastole. S3 sounds are normally inaudible and heard during the early diastole for normal children and young adults [3]. It represents diastolic overload or dysfunction of the ventricle or heart valves in older adults.

\subsection{S4 Sound}

It is caused by the ventricular filling due to atrial contraction. Abnormality in S4 represents ventricular hypertrophy, pulmonary arterial hypertension and pulmonary stenosis [3]

\section{METHODS AND METHODOLOGY}

The system consists of mainly a transmitter section Figure 1 and a receiver section Figure 2 : (a) sensor, preamplifier, and band pass filter and wirelessly transmitted by a transmitter (b) Receiver module receives data wirelessly[4].

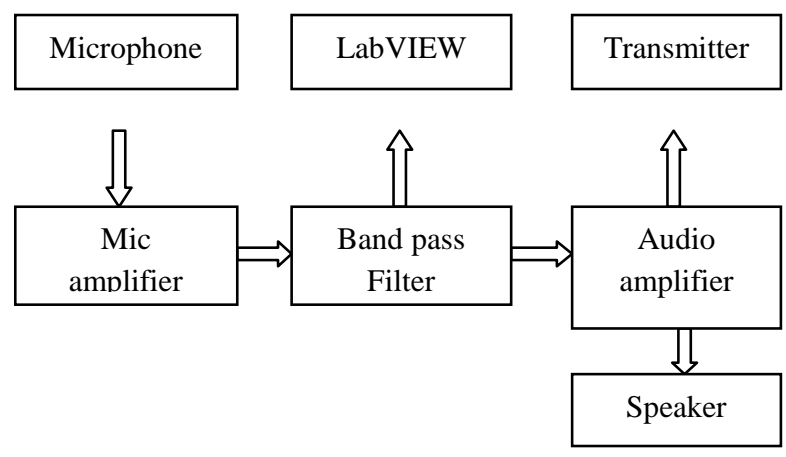

Fig 1: Proposed system transmitter section

The heart sound is picked from the body using stethoscope and an electrets microphone [5]. Then signal is amplified [6] and filtered to remove the noise and other sounds such as lung sounds, noise from the surroundings etc. This signal can then be viewed in Lab VIEW for real time monitoring of heart sound and classification as normal and abnormal depending upon beats per minute(bpm).If bpm is less than 60 it can be classified as a abnormal heart sound as bradycardia. If bpm is greater than $100 \mathrm{bpm}$ it is known as tachycardia. And heart beats per minute in between 60 and 100 is classified as normal. The output from the filter is fed to a low power audio amplifier [7]. This power amplifier is capable of driving the speaker. The power amplified signal is then transmitted using a FM transmitter module. The transmitted heart sound signal is received by a FM receiver kept at a distance. Then after signal conditioning the heart sound can be heard through a head phone or can be viewed in LabVIEW. Speakers are not recommended to use since it can form positive feedback and hence there is chance of amplification of noise. Hence use headphones are recommended instead of a speaker.

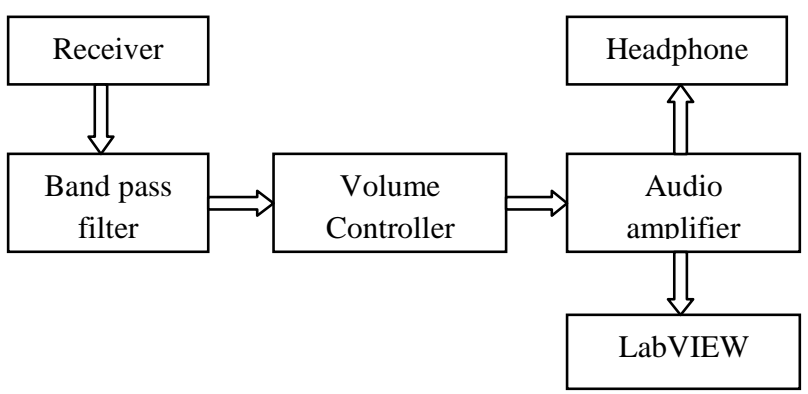

Fig 2: Proposed system Receiver section

The LabVIEW analysis is done on the heart sounds via the microphone of the laptop. In LabVIEW heart sounds are classified as bradycardia and tachycardia.

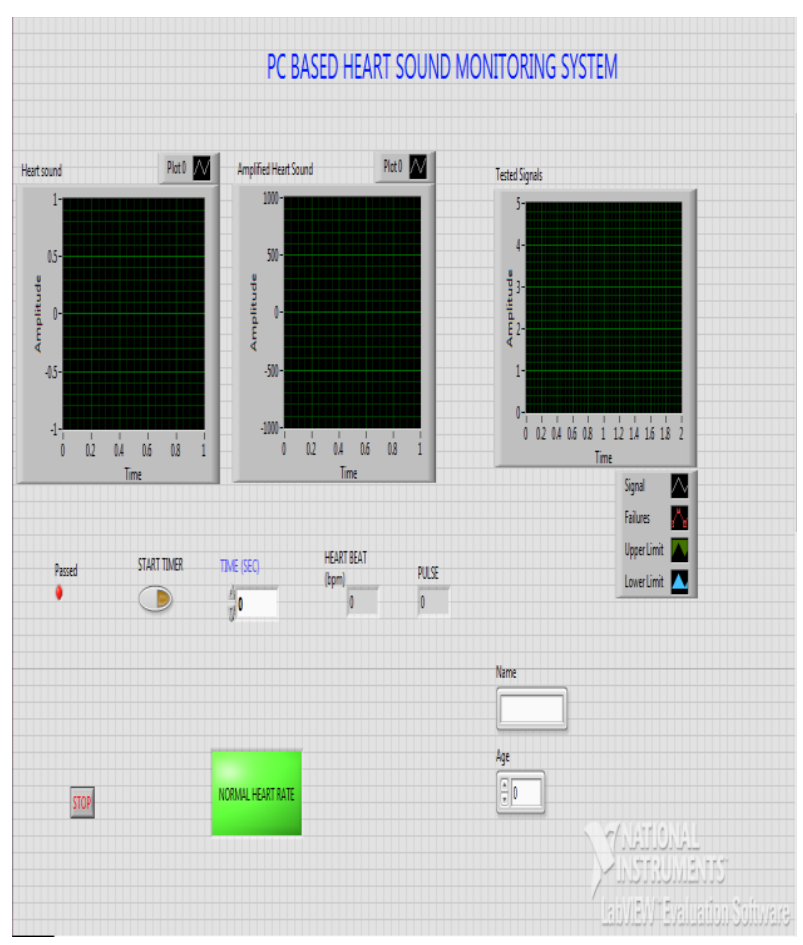

Fig 3: Design of Front Panel in LabVIEW

And for increasing the diagnosing capability of the system further classification study is being performed using MATLAB and Naive Bayer's classifiers. Classification study of heart sound is done as artifact, murmur, extra systole and normal by extracting features in MATLAB. For classification 
of heart sounds in to these four classes, different classifiers such as Naïve bayes classifier, Decision tree, Multilayer Perceptron, Ada BoostM1 etc can be used. In this work Naïve bayes classifier and decision tree classifier is used. The classification accuracy can be increased by using different classifier, taking more datasets or by extracting more features.

\section{RESULTS AND DISCUSSIONS}

The system proposed has been successfully implemented in effective monitoring and diagnosing of heart sound abnormalities. Real time acquisition of the patient heart sound has been possible with minimum noise. The heart sound is picked using a stethoscope collected from dispensary and an electrets microphone. Then signal is amplified and filtered using an instrumentation amplifier of gain $1000 \mathrm{~V} / \mathrm{V}$ and a band pass filter with a cut off frequencies of $20-1500 \mathrm{~Hz}$. This signal can be recorded using AUDACITY software and can be stored as a dataset. This software records the heart sound and displays it when required

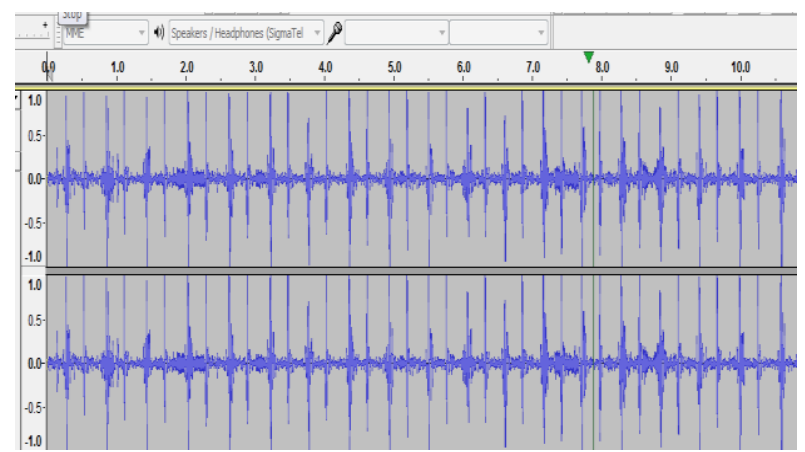

Fig 4: PCG of an individual obtained in real time using AUDACITY.

The real time acquired heart sound signal in Lab VIEW from preprocessing stages such as instrumentation amplifier, Butterworth wide band pass filter and audio amplifier is depicted in Figure 5. Y scale gives the amplitude of the signal in Volt $(\mathrm{V})$ and $\mathrm{X}$ scale gives the time in seconds (sec).

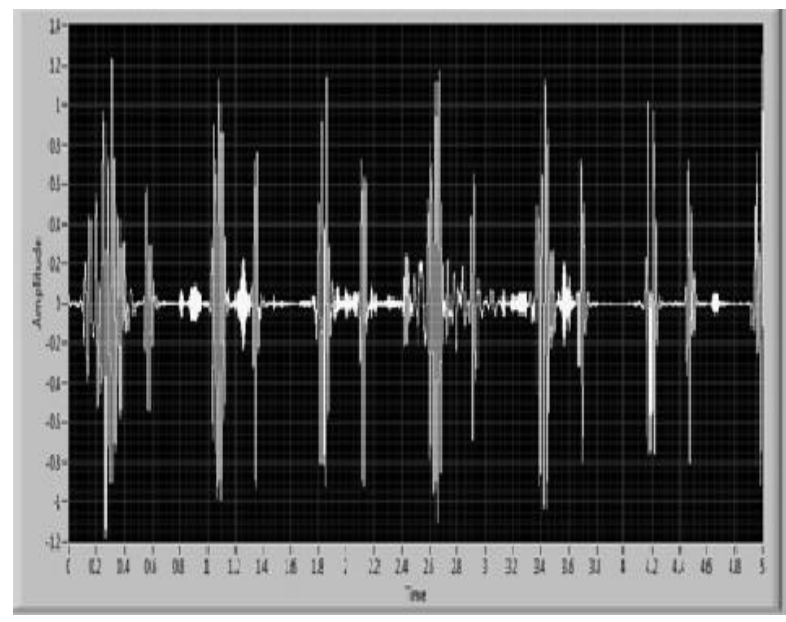

Fig 5: Real time heart sound acquisition in Lab VIEW

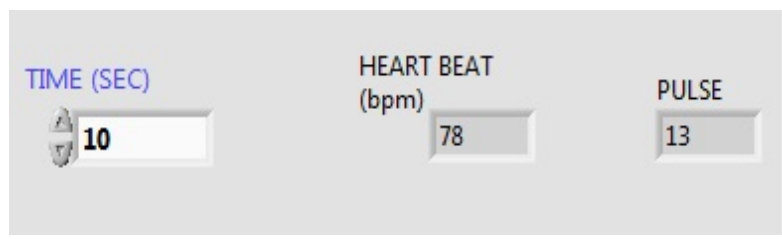

Fig 6: Display beats parameters

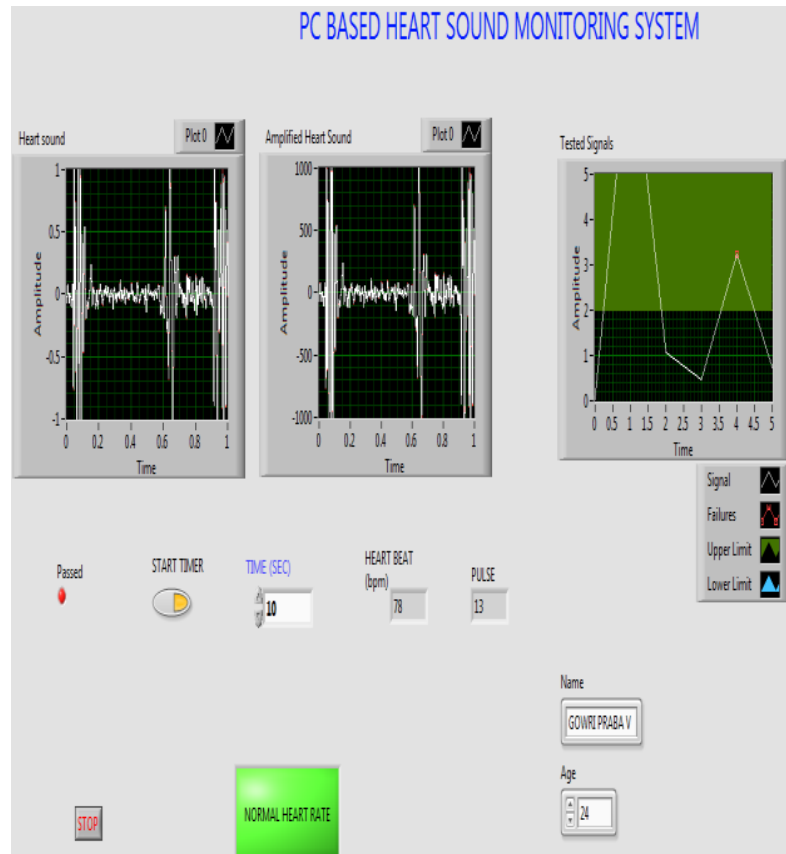

Fig 7: Front panel of PC Based Heart Sound Monitoring System

Finally in this work a study has been conducted to study how to classify the real heart sound audio which is also known as "beat classification" into one of four categories as Normal, Murmur, Extra Heart Sound and Artifact. A dataset, which contains many files in WAV format for murmur, extra systole, artifact and normal, was chosen. The audio files are of varying lengths, between 1 second and 30 seconds and some have been clipped to reduce excessive noise and provide the salient fragment of the sound. When an FFT study of these where conducted it has been observed that most information in heart sounds is contained in the low frequency components, with noise in the higher frequencies. Hence it is common to apply a low-pass filter at $195 \mathrm{~Hz}$. Fast Fourier transforms are also likely to provide useful information about volume and frequency over time of each classes [8]. Initially, features such as FFT, standard deviation, mean, median, mode, skewness, and kurtosis are extracted from the database. The database consists of heart sounds of four different classes they are murmurs, extra systole, artifact, and normal. Initially these values of each files are then stored separately. FFT's of each file are also stored separately. Then plotted FFT's and find that plot is different for murmur, extra systole, artifact and normal. Then classification study is performed using different classifiers.

Table 1 shows the confusion matrix obtained by using Naïve Bayes classifier [9]. Here features extracted are mean, median, mode, skew and Kurt. The classes for classification are murmurs, extra systole, artifacts and normal. The number of samples for each class chosen is different. 'a' represents murmurs and out of 19 murmurs 14 murmurs are correctly classified as murmurs. ' $b$ ' represents extra systole and among 19 extra systole 13 extra systole are correctly classified. 'c' represents artifacts and out of 23 artifacts, 3 artifacts are correctly classified as artifacts. Finally represents normal and among 19, 7 normal data's are correctly classified as normal. 
Table 1. Confusion matrix of Naïve Bayes classifier with an accuracy of $46.25 \%$

\begin{tabular}{|c|c|c|c|c|}
\hline & $\mathrm{a}$ & $\mathrm{b}$ & $\mathrm{c}$ & $\mathrm{d}$ \\
\hline $\mathrm{a}$ & 14 & 4 & 0 & 1 \\
\hline $\mathrm{b}$ & 6 & 13 & 0 & 0 \\
\hline $\mathrm{c}$ & 6 & 8 & 3 & 6 \\
\hline $\mathrm{d}$ & 5 & 7 & 0 & 7 \\
\hline
\end{tabular}

Table 2 shows the confusion matrix obtained by using Naïve Bayes classifier. Here features extracted are mean, median, mode, skew and Kurt. The classes for classification are murmurs, extra systole, artifacts and normal. The number of samples for each class chosen is different. 'a' represents murmurs and out of 19 murmurs 13 murmurs are correctly classified as murmurs. ' $b$ ' represents extra systole and among 19 extra systole 17 extra systole are correctly classified. 'c' represents artifacts and out of 19 artifacts, only 1 artifact is correctly classified as artifact. Finally represents normal and among 19, 6 normal data's are correctly classified as normal.

Table 2. Confusion matrix of Naïve Bayes classifier with an accuracy of $\mathbf{4 8 . 6 8 \%}$

\begin{tabular}{|c|c|c|c|c|}
\hline & $\mathrm{a}$ & $\mathrm{b}$ & $\mathrm{c}$ & $\mathrm{d}$ \\
\hline $\mathrm{a}$ & 13 & 6 & 0 & 0 \\
\hline $\mathrm{b}$ & 2 & 17 & 0 & 0 \\
\hline $\mathrm{c}$ & 2 & 11 & 1 & 5 \\
\hline $\mathrm{d}$ & 3 & 9 & 1 & 6 \\
\hline
\end{tabular}

Table 3 shows the confusion matrix obtained by using Naïve Bayes classifier. Here features extracted are mean, median, mode, skew and Kurt. The classes for classification are murmurs, extra systole, artifacts and normal. The number of samples for each class chosen is different. ' $a$ ' represents murmurs and out of 33 murmurs 28 murmurs are correctly classified as murmurs. 'b' represents extra systole and among 19 extra systole 11 extra systole are correctly classified. 'c' represents artifacts and out of 40 artifacts, 7 artifacts are correctly classified as artifacts. Finally represents normal and among 31, 7 normal data's are correctly classified as normal.

Table 3. Confusion matrix of Naïve Bayes classifier with an accuracy of $43.2 \%$

\begin{tabular}{|c|c|c|c|c|}
\hline & $\mathrm{a}$ & $\mathrm{b}$ & $\mathrm{c}$ & $\mathrm{d}$ \\
\hline $\mathrm{a}$ & 28 & 4 & 1 & 0 \\
\hline $\mathrm{b}$ & 7 & 11 & 0 & 1 \\
\hline $\mathrm{c}$ & 18 & 8 & 7 & 7 \\
\hline $\mathrm{d}$ & 15 & 8 & 1 & 7 \\
\hline
\end{tabular}

Table 4 shows the confusion matrix obtained by using Decision tree classifier. Here features extracted are mean, median, mode, skew and Kurt. The classes for classification are murmurs, extra systole, artifacts and normal. The number of samples for each class chosen is different. ' $a$ ' represents murmurs and out of 33 murmurs 28 murmurs are correctly classified as murmurs. 'b' represents extra systole and among 19 extra systole 11 extra systole are correctly classified. 'c' represents artifacts and out of 40 artifacts, 7 artifacts are correctly classified as artifacts. Finally represents normal and among 31, 7 normal data's are correctly classified as normal.

Table 4. Confusion matrix of Decision Tree classifier with an accuracy of $50.2 \%$

\begin{tabular}{|c|c|c|c|c|}
\hline & $\mathrm{a}$ & $\mathrm{b}$ & $\mathrm{c}$ & $\mathrm{d}$ \\
\hline $\mathrm{a}$ & 28 & 4 & 1 & 0 \\
\hline $\mathrm{b}$ & 7 & 11 & 0 & 1 \\
\hline $\mathrm{c}$ & 18 & 8 & 7 & 7 \\
\hline $\mathrm{d}$ & 15 & 8 & 1 & 7 \\
\hline
\end{tabular}

Classification accuracy is not the best but can be increased by taking more features and taking different classifiers.

\section{CONCLUSION}

A PC based heart sound monitoring system with low cost has been designed. It offers the advantage of electronic stethoscope, digital stethoscope and recording stethoscope. The heart sounds from different people are recorded using the hardware setup and Lab VIEW. The obtained heart sound can be transmitted from one end to other using FM modulator used in cars and can be received at other ends with FM receivers. Then classification results based on bpm is obtained in LabVIEW 2010. Arrhythmias' such as tachycardia and bradycardia can be detected with the help of LabVIEW. The system has been tested on 15 individuals to display their beats per minute For Further classification of heart sound as murmur, extra systole, artifact and normal, studies with different numbers of data samples for each class, different classifiers, different features etc are made use. This classification when integrated with system increases its diagnosing capabilities. At the same time the cost of the system is very less since it requires cheaper and fewer components.

\section{FUTURE SCOPE}

The system could be made more user friendly and portable by incorporating the system with a microcontroller such as MSP430.The classification accuracy is only $50.4 \%$, but can be increased in future by the use of a different classifier or extracting different features. The classification study done using MATLAB could be sinked into the PC based Heart Sound Monitoring System for effective treatment procedure. Further classification studies on different valves disorders and their abnormal heart sounds can be done in future in order to increase the diagnosing capability of the physician.

Heart sounds gives valuable diagnostic information needed for early detection of cardiovascular defects and diseases and in future could also be used in other applications such as biometrics in which a person can be identified from their unique heart sound signal. 


\section{ACKNOWLEDGMENTS}

Our thanks to Dr. Madhu Mohan, coordinator of MTech Biomedical Engineering in Amrita Vishwa Vidyapeetham, Coimbatore for providing us support and guidance though he had busy schedule managing the classes. We owe our profound gratitude to $\mathrm{N}$ Premachandran and our guide Mr. Binoy B. Nair who guided us the long way for the completion of our work by providing all the necessary information for converting an idea into a reality.

\section{REFERENCES}

[1] R.S.Khandpur, 1989. Handbook of biomedical instrumentation, second edition Tata McGraw-Hill Publishing Company Limited, NewDelhi, pp no. 167170.

[2] M. S. Manikandan and K. Soman, 2010. Robust heart sound activity detection in noisy environments, Electronics letters, vol. 46, pp no. 1100-1102.

[3] D.Balasubramaniam,D.Nedumaran, 2010. Efficient Computation of Phonocardiographic Signal Analysis in
Digital signal processor Based System, International journal of computer theory and Engineering, vol.2, No.4.

[4] Mihir Vaidya,Snehal Mhatre, Madhuri Ware, Pratik Pradhan, 2013. Embedded stethoscope, International Journal of Emerging Technology and Advanced Engineering, Vol 3, Issue 2.

[5] Diagnostics, monitoring and therapy: Digital stethoscopes.[online].Available:http://tinyurl.com/m4lrw as, [Last Accessed: 20 August 2012].

[6] Franco, S. 1988. Design with operational amplifiers and analog integrated circuits: McGraw-Hill New York.

[7] Kamichik, S. 1999. Designing Power Amplifiers. Prompt Publications.

[8] Li, Tan. and Jiang, J. 2013.Digital signal processing: fundamentals and applications: [Access Online via Elsevier].

[9] Rish, I. 2001. An empirical study of the naive Bayes classifier, in IJCAI 2001 workshop on empirical methods in artificial intelligence. pp. 41-46. 